\title{
Adaptive Corrosion Protection System Using Continuous Corrosion Measurement, Parameter Extraction, and Corrective Loop
}

\author{
Jasbir N. Patel, Andre Chang, Haleh Shahbazbegian, and Bozena Kaminska \\ School of Engineering Science, Simon Fraser University, 8888 University Drive, Burnaby, BC, Canada V5A 1S6 \\ Correspondence should be addressed to Jasbir N. Patel; jpatel@sfu.ca
}

Received 27 October 2015; Accepted 24 February 2016

Academic Editor: Michael J. Schütze

Copyright (C) 2016 Jasbir N. Patel et al. This is an open access article distributed under the Creative Commons Attribution License, which permits unrestricted use, distribution, and reproduction in any medium, provided the original work is properly cited.

\begin{abstract}
A simple current-sourced adaptive corrosion protection system (ACPS) along with a technology to extract the protection current from the Tafel plot is presented. For reliable protection of the target metal, first, the Tafel plot of the target metal is obtained. Subsequently, a novel technique proposed in this paper is used to extract the protection current from the Tafel plot. This extracted protection current is fed to the target metal to protect the metal in the existing corrosive environment. This threepart system is adaptively used to update the required protection current to effectively protect the target metal continuously. All these functionalities are integrated in a stand-alone ACPS that effectively diagnoses the corrosion status and updates the protection parameters without any manual interaction or physical modification of the set-up to offer modularity, reliability, and cost saving. To validate the technique, a laboratory scale system is realized and tested using various metal samples and various corrosive mediums. Using the experimental system, A36 metal coupons are effectively protected with protection (inhibition) efficiency of 40-100\% in different corrosive mediums that can extend the life expectancy of the target metal from $\sim 2$ times to more than 100 times for the tested corrosive mediums.
\end{abstract}

\section{Introduction}

Despite being a well-known problem, corrosion of structures continues to be a large expense in different industries such as pipelining, aeronautics, and basic infrastructures. The corrosion of structures such as concrete bridges [1-3], concrete girders [4], and concrete structures in a marine environment [5] compromises safety and functionality of many infrastructures, leading to a costly repair [6]. Amongst other things, environmental factors such as environmental resistivity, humidity, exposure to electrolytes, and $\mathrm{pH}$ all play a key role in the rate at which a material such as ship structures [7], stainless steel [8], galvanized steel [9], and carbon steel [10] will corrode.

Corrosion is defined by the National Association of Corrosion Engineers (NACE) [11] as the naturally occurring deterioration of a material (usually a metal) which results from a chemical or electrochemical reaction [12] with its environment. A simple corrosion theory [13] and a physical model of the corrosion [14] are also published previously. Despite the thermodynamic tendency to undergo the oxidation reaction, there are time-proven methods that prevent and control corrosion which can reduce or eliminate its impact on public safety, economy, and environment $[15,16]$. Many techniques have been developed over the years in order to protect a targeted metal. Some popular corrosion prevention techniques include use of an organic metal [17], a mechanical technique [18], a cathodic protection [19], and a photoelectrochemical approach [20]. Besides, uses of different coatings $[21,22]$ and electrochemical protections have proven to extend lifetime of the target metal in corrosion prone environments.

Popular methods include the use of cathodic protection systems $[19,23]$, one of which uses target metal as a cathode that is protected by the use of a sacrificial metal that acts as an anode (Galvanic). The impressed current cathodic protection 
(ICCP) system is well explained and studied over decades $[23,24]$. The effect of the alternating current $(\mathrm{AC})$ for the ICCP system [25] and use of the ICCP system to protect a costal bridge structure [26] has also been described in previous studies. Though an initial installation of an ICCP system [23] is more costly, it has proven to be a cost-effective method of protection since it does not require the systematic replacement of a degraded anode.

In a typical ICCP system, appropriate control of the protection current is very important [27]. The overprotection (too much cathodic protection current) of the metal creates undesired potential throughout the structure and leads to an acceleration of the corrosion process [28]. Additionally, inappropriate ICCP system can also degrade bond strength in concrete structures [29] or the protective coating [30]. Hence, corrosion engineers must constantly evaluate and monitor existing corrosion status.

For example, in order to protect a steel structure, it has been standardized that $-0.85 \mathrm{~V}$ (versus $\mathrm{Cu} / \mathrm{CuSO}_{4}$ reference electrode) be applied [31]; however, this potential is compensated by the instant-off potential measured in the field environment $[32,33]$. However, such instant-off potential measurements are done manually in a field environment, which requires manual involvement even in remote areas. Hence, effectiveness of the corrosion protection is highly dependent on the human interaction, which is neither efficient nor costeffective [34,35]. Besides, capacitive spikes that appear during the current interruption can mask the instant-off potential $[36,37]$. Hence, the instant-off potential measurement and the existing potential-based corrosion protection system have many limitations that should be addressed to achieve lowcost, reliable, and automated corrosion protection system. Because of the target metal properties and other environmental factors, some localized corrosion may occur in a metal structure. Effects of such nonuniform corrosion on the cracking and service life of the concrete structures have been investigated previously [38]. Even mathematical model to evaluate uniform and nonuniform corrosion induced damage in reinforced concrete is also developed [39]. Therefore, the nonuniform corrosion distribution or protection in a metal structure is a challenging problem to address.

To address these limitations, we propose a simple adaptive corrosion protection system (ACPS) to reliably protect the target metal in a wide variety of environments. The ACPS consists of measuring the electrochemical response of the target metal to obtain Tafel plot, extracting the exact protection current from the Tafel plot and adjusting the required current by using the feedback loop. This results in a diagnostic as well as protection system that can accurately monitor and protect the target metal. The feedback loop automatically measures the electrochemical behaviour (Tafel plot) analytically at the user-defined intervals and protects the target metal based on the existing corrosive conditions. The proposed closed-loop corrosion protection system offers higher reliability and precision without any adverse effects mentioned earlier. Additionally, the manual interactions are completely eliminated in the proposed system. The proposed system also optimizes the energy requirement and reduces the power waste which results in energy cost savings.

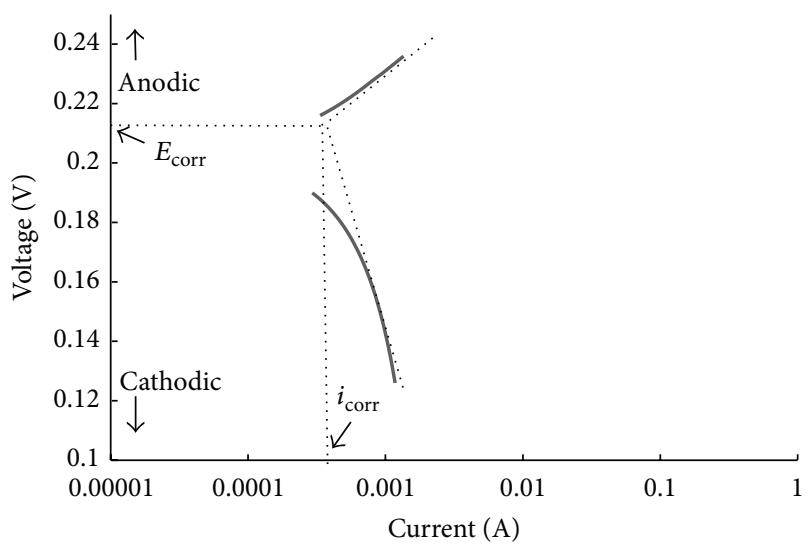

FIGURE 1: Polarization curves for iron in a quiescent solution of $0.6 \mathrm{M} \mathrm{NaCl}$ open to the air. The anodic and cathodic branches are pointed in the plot. In addition, the slopes of the anodic and cathodic branches along with interpolation of the two branches are also extracted using the dotted line. Based on this extraction, the $E_{\text {corr }}$ and $i_{\text {corr }}$ values are also determined.

\section{Theory of the ACPS}

In a typical corrosion measurement system, electrochemical response of the target metal in a corrosive environment is obtained using an electrochemical cell and the Tafel plot (see Figure 1) is obtained from the electrochemical response. The values of open-circuit potential $\left(E_{\text {corr }}\right)$ and the corrosion current $\left(i_{\text {corr }}\right)$ (see Figure 1 ) are typically extracted from the Tafel plot to calculate corrosion rate of the target metal in the given corrosive medium [40]. Additionally, the Tafel plot is accurately used to measure corrosion rate of different metals [41] and the reinforced steel [42].

It is important to note that the potential for this $i_{\text {corr }}$ is usually the same as or very close to the open-circuit potential $\left(E_{\text {corr }}\right)$ and $i_{\text {corr }}$ value obtained from the Tafel plots is also the natural current flow created in a given electrochemical cell when both the oxidation and the reduction reactions are in equilibrium [43]. Hence, at this current $\left(i_{\text {corr }}\right)$ and potential $\left(E_{\text {corr }}\right)$, metal should be neither underprotected (not sufficient protection current or potential) nor overprotected. Thus, it is the minimum amount of current required to protect the metal from freely corroding in the present corrosion set-up. Using this minimum amount of current $\left(i_{\text {corr }}\right)$, the optimum protection parameters can be determined by the remaining amount of corrosion that is acceptable. The proposed method opens the opportunity to control the protection current as desired by the corrosion engineer. In combination with the open-circuit voltage $\left(E_{\text {corr }}\right)$ it is possible to predict the minimum amount of power required to protect a targeted metal. However, as we can see from the Tafel plot, the actual potential at current $i_{\text {corr }}$ is slightly shifted towards the cathodic region from the $E_{\text {corr }}$ potential, which ensures effectiveness of the corrosion protection at the $i_{\text {corr }}$ current.

In summary, it is possible to precisely extract the optimum protection parameters for the present environmental condition of the target metal using the technique described 


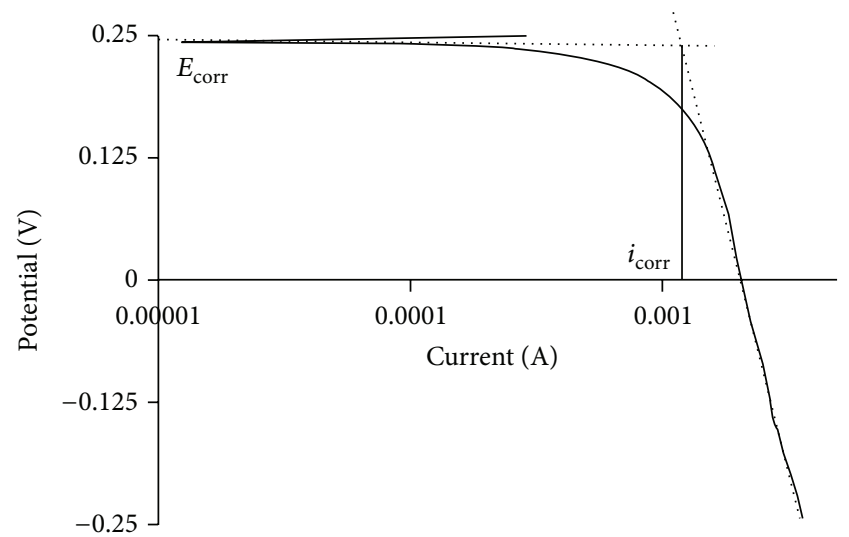

FIGURE 2: The simplified system of $i_{\text {corr }}$ extraction. The linear part of the cathodic region is extended until it reaches the $E_{\text {corr }}$ potential to determine $i_{\text {corr }}$ value.

above. Also, the values of these optimum protection parameters reflect the changing environmental conditions as well as the metal corrosion status if frequent measurements can be done without changing the protection set-up. By adapting to this updated protection parameters, it is possible to protect the target metal more scientifically without the risk of underprotection or overprotection.

In a further analysis, during the electrochemical measurement (Tafel plot) of the anodic branch (Tafel plot), the target metal oxidizes slightly and hence corrodes during the anodic sweep. Even though this corrosion amount is minimal, the optimum protection parameters deviate slightly after completion of the test. This results in a slight underprotection for the optimally protected target metal. To eliminate this error, the proposed system is further simplified to minimize this anodic sweep from the electrochemical measurement to increase accuracy, reliability, and performance over an extended period (see Figure 2).

As shown in Figure 2, the electrochemical sweep is started from the anodic branch which is slightly higher than the open-circuit potential in the improved method. In this method, the value of $i_{\text {corr }}$ is determined by extrapolating linear region of the cathodic branch and the open-circuit potential $\left(E_{\text {corr }}\right)$ (see Figure 2 ). By using this method, the $E_{\text {corr }}$ and $i_{\text {corr }}$ values are accurately determined similar to the earlier method and reduce the small amount of metal corrosion because of the anodic sweep. This method also reduces the overall time required to measure the full behaviour and parameter extraction.

By adapting (feedback loop) the proposed currentsourced corrosion protection system and the extracted corrosion protection parameters $\left(i_{\text {corr }}\right.$ and $\left.E_{\text {corr }}\right)$, the target metal can be accurately protected with the precise amount of the corrosion protection. Unlike a classical potential-based ICCP system, the proposed method avoids overprotection or underprotection of the target metal and hence the adverse effects associated with the same. In our proposed technique, the electrochemical behaviour of the target metal is measured frequently and the optimum protection parameters

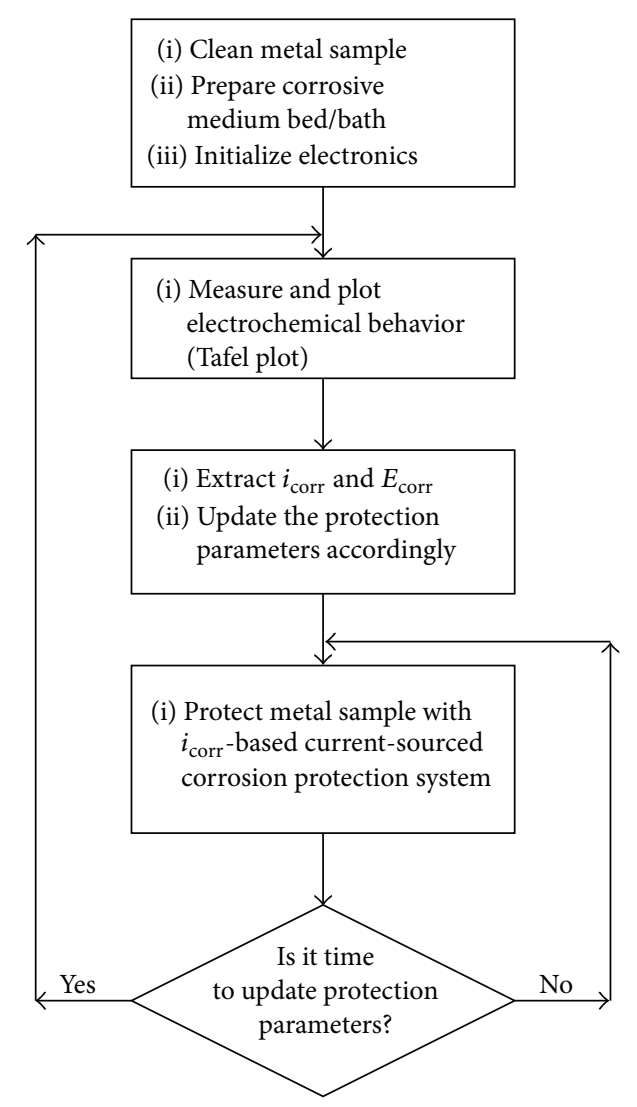

FIGURE 3: Flow chart of the proposed adaptive corrosion protection system (ACPS).

are extracted. As soon as the new optimum protection parameters are extracted, they are adapted in the protection system until the new measurement and extractions are done. We also propose to use a current-sourced protection system to effectively protect the target metal with the optimum power requirement.

\section{The Proposed Adaptive Corrosion Protection System (ACPS)}

The ACPS offers a novel approach over the classical potentialsourced corrosion protection system by proposing the full feedback loop based adaptive corrosion protection system.

As described in the previous section, the ACPS consists of a simple $i_{\text {corr }}$-based current-sourced corrosion protection system, which monitors the corrosion status at user-defined intervals and protects the target metal by adapting to the change in the corrosion status of the protected metal. Hence, the ACPS control module works as an active feedback loop system to update the protection current that is extracted from the electrochemical sweep of the protected system.

The flow chart of the ACPS is shown in Figure 3 and the exact execution steps are described as follows:

(1) The implementation of the ACPS (Figure 3) starts with the preparation of the metal to be protected and initialization of the control unit. After the initial 


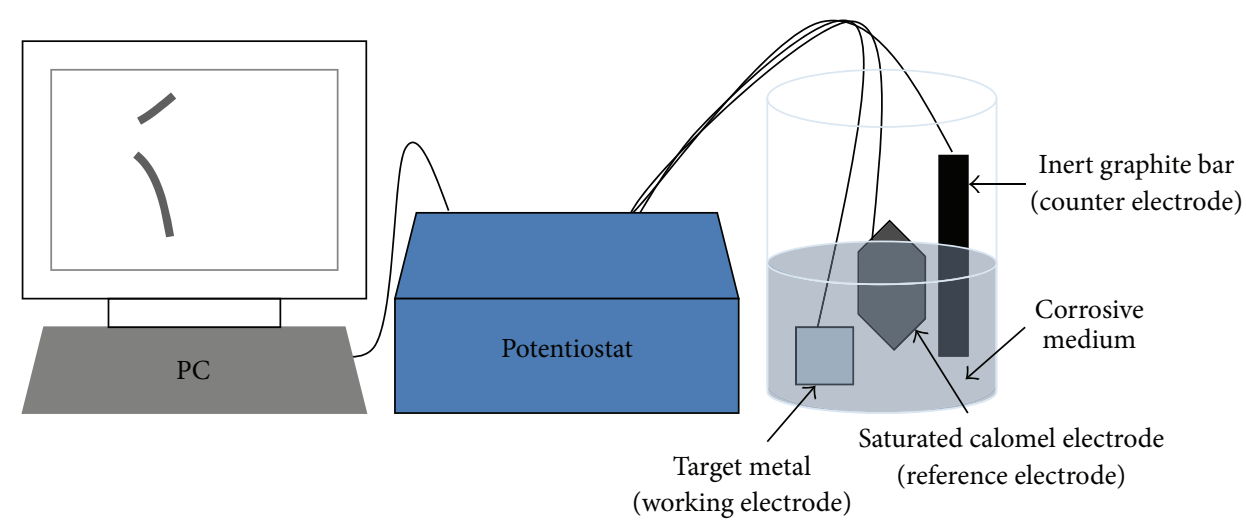

FIgURE 4: The experimental set-up to test the electrochemical behaviour.

preparation is done, the target metal and the control unit are connected together. The interval to update the protection parameters is set at this time. However, this interval can be easily updated during execution.

(2) As soon as the test parameters are determined, the electrochemical measurement is performed and the Tafel plot of the target metal is obtained.

(3) The value of $i_{\text {corr }}$ is then extracted from the Tafel plot and the protection current is updated to adapt to changes in the corrosive state of the target metal.

(4) The metal is continuously protected with the protection current $\left(i_{\text {corr }}\right)$ extracted in step (3) until the time interval to update the protection parameters is reached.

(5) When the time interval to update the protection parameters is reached, the complete cycle is started again from step (2).

We can see from the flow chart (Figure 3) and the steps above that the proposed control unit can be realized using a stand-alone embedded module, a LabVIEW based virtual instrument, or other low-cost systems. Additionally, a lowcost graphite bar can also be used as an electrode. Hence, the overall implementation of the proposed ACPS can be very cost-effective and it is simple to implement in a laboratory or a field environment. The ACPS system can also be used as a diagnostic tool to determine exact corrosion state of the target metal.

\section{Materials and Methods}

To prove the functionality of the ACPS and ability to protect a target metal effectively, systematic experiments are carried out in a laboratory environment. Each individual experimental set-up is described below with specific details and the appropriate block diagram.

4.1. Experimental Set-Up for Electrode Configuration. To determine usability of the electrochemical set-up for the ACPS, a set of experiments is established using a personal computer (PC) controlled potentiostat (ParStat 4000 from Princeton Applied Research) (see Figure 4).

The typical electrochemical set-up (Figure 4) is implemented by connecting the target metal to the working electrode, an inert graphite bar to the counter electrode, and the saturated calomel electrode to the reference electrode of the potentiostat. The experimental set-up is prepared using a $0.6 \mathrm{M} \mathrm{NaCl}$ solution as the corrosive medium and an A36 hot-rolled steel sample $(2.5 \mathrm{~cm} \times 2.5 \mathrm{~cm} \times 0.5 \mathrm{~cm})$ as the target metal. Hence, the surface area exposed to the corrosive medium during experiments is $3125 \mathrm{~mm}^{2}$.

The Tafel plots are obtained by sweeping potential on the working electrode with respect to the reference electrode for the given three-electrode configuration. For the experiments, the potential is swept from $-0.25 \mathrm{~V}$ to $0.25 \mathrm{~V}$ with the scan rate of $2 \mathrm{mV} / \mathrm{sec}$. The standard calomel electrode (SCE) is used as the reference electrode for all the measurements. The A36 steel sample is cleaned before each experiment to achieve repeatability in the measurements. Once complete, the $i_{\text {corr }}$ value is extracted for each experiment and analyzed.

4.2. Experimental Set-Up for Corrosion Protection. After understanding behaviour of the electrochemical measurement and the resultant Tafel plot, experimental validation of the current-sourced protection system is performed. The extracted $i_{\text {corr }}$ value is used as the protection current for these experiments.

To validate the principle, two different metal samples, a common galvanized hardware washer (surface area: $278.5 \mathrm{~mm}^{2}$ ) and an A36 steel sample (same as previous experiment), are used. Before starting the experiment, the metal samples are polished and rinsed using isopropyl alcohol (IPA) and deionized (DI) water and dried under the $\mathrm{N}_{2}$ stream. Two separate samples of the washer and the A36 steel are prepared. One washer and an A36 steel sample are protected using the proposed system and the second set is left in the corrosive medium to corrode freely. The $0.6 \mathrm{M} \mathrm{NaCl}$ solution is used as the corrosive medium. To extract the $i_{\text {corr }}$ current, Tafel plot is obtained using the set-up described in Figure 4 . The $i_{\text {corr }}$ value is extracted using the potentiostat software and directly used to feed the current as a currentsourced corrosion protection system. The first set with both 
TABLE 1: Different corrosive mediums used for the validation of the proposed ACPS. The concentrations of the corrosive mediums if they are important and the importance of each corrosive medium are also described.

\begin{tabular}{|c|c|c|}
\hline Name of the corrosive medium & $\begin{array}{l}\text { Concentration of the corrosive medium, } \\
\text { if applicable }\end{array}$ & Comment \\
\hline Sink water & - & $\begin{array}{l}\text { Typical tap water in North America, } \\
\text { which is quite corrosive }\end{array}$ \\
\hline Humic acid & $50 \mathrm{mg} / \mathrm{L}$ & $\begin{array}{l}\text { It is a known substance to cause } \\
\text { corrosion in the agricultural lands }\end{array}$ \\
\hline Wet soil bed & - & This is a highly corrosive environment \\
\hline Dry sand bed & - & This is a very weak corrosive environment \\
\hline
\end{tabular}

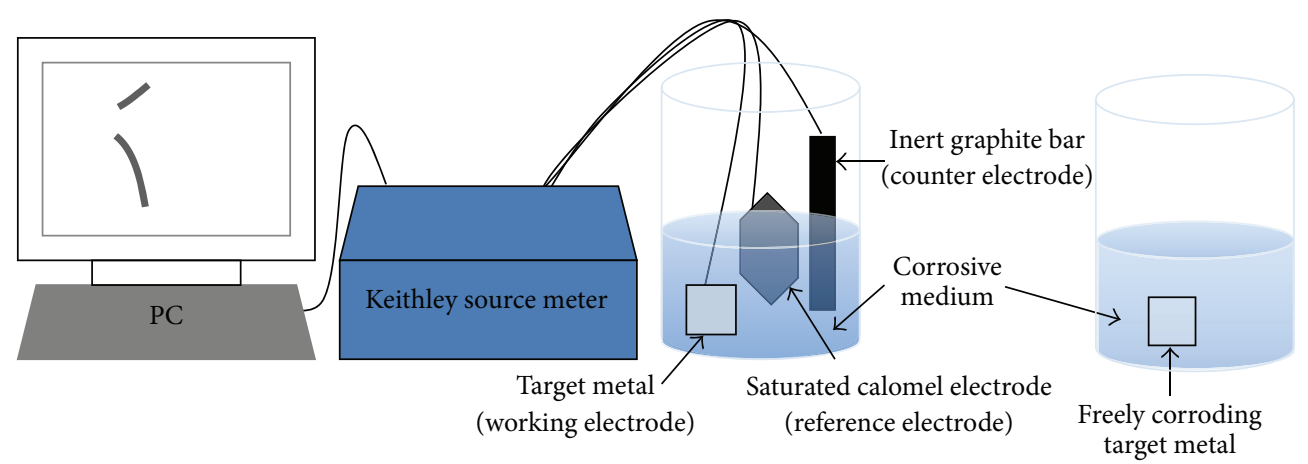

FIGURE 5: A simple experimental set-up to validate the proposed adaptive loop. A simple and cost-effective DC source meter controlled with the LabVIEW ${ }^{\circledR}$ VI is used for the set-up.

metal samples is protected for 24 hours for the proof-ofconcept test. The other set is corroded freely for the same duration before all the samples are cleaned in an IPA bath and weighted again to determine weight loss of the samples.

To further validate the proposed corrosion protection system, the same experiment is extended for more than two weeks with weight measurements, electrochemical measurements, $i_{\text {corr }}$ extraction, and current-source modification every 24 hours. The weight loss for all the samples is then analyzed. Protection (inhibition) efficiency of the system is then calculated using the following equation [44]:

$$
\eta(\%)=\frac{W_{\text {freely corroded }}-W_{\text {protected }}}{W_{\text {freely corroded }}} \times 100,
$$

where $\eta(\%), W_{\text {freely corroded }}$, and $W_{\text {protected }}$ represent protection (inhibition) efficiency, weight loss of the freely corroded metal sample, and weight loss of the protected metal sample, respectively.

4.3. The Adaptive Loop Set-Up. After validating the functionality of the $i_{\text {corr }}$-based current-sourced corrosion protection system, an automated and simple system is implemented using a simple DC source meter (Keithley Model 2400) controlled by a personal computer-based stand-alone ACPS that is implemented using the National Instrument (NI) LabVIEW based VI (see Figure 5).

In this low-cost set-up, the A36 steel sample $(2.5 \mathrm{~cm} \times$ $2.5 \mathrm{~cm} \times 0.5 \mathrm{~cm})$ is used for all the experiments. First, the $0.6 \mathrm{M} \mathrm{NaCl}$ solution is used as the corrosive medium. Additionally, this experiment is performed using one A36 sample protected with the automated adaptive loop and the other A36 sample is corroded freely. The weight measurements and data collection method are the same as those in the previous subsection.

The LabVIEW VI is configured to sweep the Tafel plot of the target metal at the user-defined interval. In our experimental set-up, the Tafel plot measurement and protection parameter extraction are done every 24 hours. The value of $i_{\text {corr }}$ is extracted from the LabVIEW VI and used by the DC source meter to protect the target metal until the $i_{\text {corr }}$ value is extracted again. This simple and portable adaptive loop based system is also configured to obtain performance indicators of the corrosion protection continuously.

4.4. Set-Up for the ACPS Performance Study in Different Corrosive Mediums. The preliminary tests to prove the concept are performed using the $0.6 \mathrm{M} \mathrm{NaCl}$ solution as the corrosive medium. However, to further validate the effectiveness of the proposed ACPS to protect the target metal, the protection should be verified in different corrosive mediums that are typically used in the field of corrosion measurement and protection (see Table 1).

The corrosive mediums for this study are selected based on the difference of their corrosive properties and ease of availability. First, to validate our proposed principle and system, readily available sink water from a kitchen tap has been used. Secondly, the $50 \mathrm{mg} / \mathrm{L}$ humic acid solution is examined. Typically, the humic acid solution is used in research to replicate the corrosive effect of the agricultural lands. After achieving promising results from the above experiments, the ACPS tests are further expanded to use 


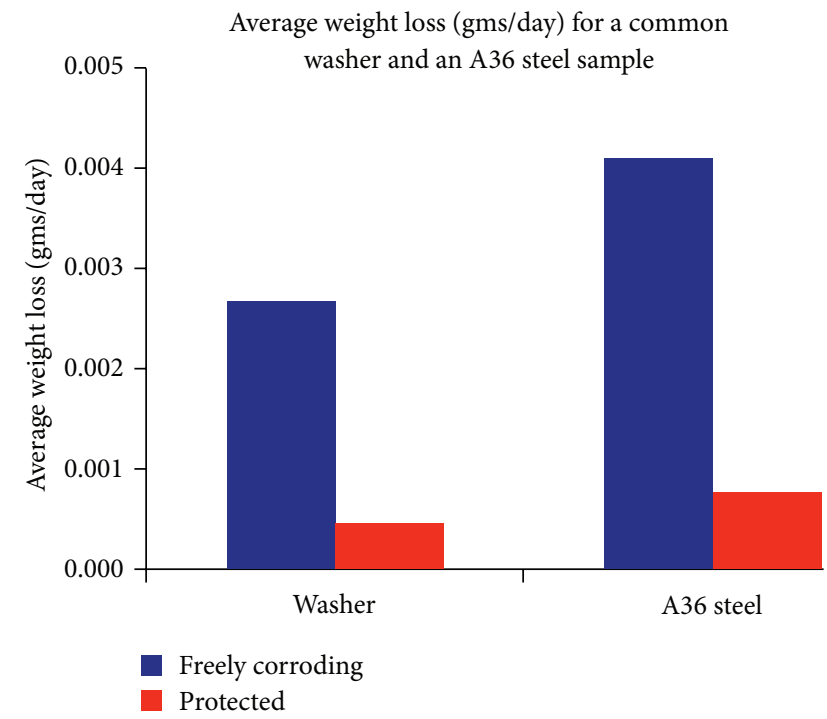

(a)

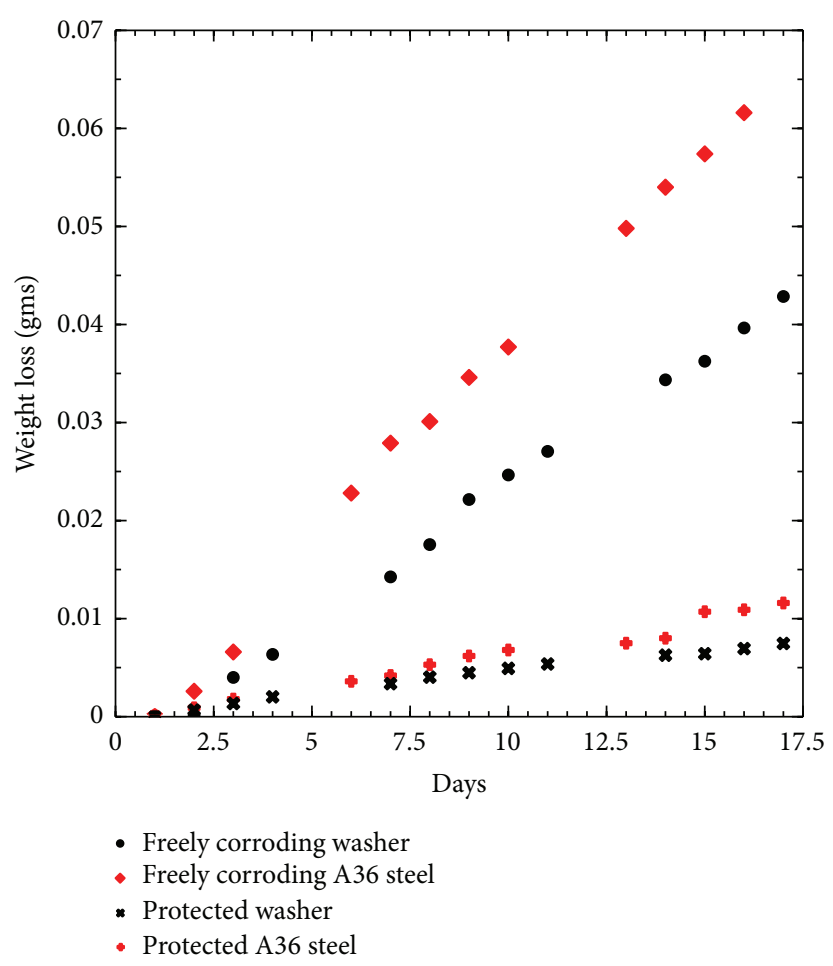

(b)

FIGURE 6: Weight loss of the freely corroding and the protected washer and A36 steel sample. Two different metal samples, a $1^{\prime \prime}$ steel washer and an A36 steel metal, are immersed in the $0.6 \mathrm{M} \mathrm{NaCl}$ solution for (a) 24 hours and (b) more than two weeks. (a) The weight loss for 24 hours and (b) the weight loss for more than two weeks.

directly available garden soil and coarse sand samples. The wet soil is highly conductive medium because of the large amount of minerals in soil. Because of this, the wet soil is considered as a highly corrosive medium. On the other hand, the dry sand is poorly conductive and hence it is typically considered as a poorly corrosive medium. The different corrosive mediums selected here present different electrochemical conditions which results in different ICCP requirements to protect the target metal.

The ACPS set-up (shown in Figure 5), analysis method, data collection, and performance analysis method for these experiments remain the same as those in the previous section.

\section{Results and Discussion}

In this section, the main results and outcome during the laboratory experiments with the ACPS are presented. As described in the previous section, the validity of the extracted $i_{\text {corr }}$ to protect different metal samples is examined first. The proof of accurate functioning of the adaptive loop is subsequently described followed by the ACPS performance assessment using the different corrosive mediums.

5.1. Validation of the Extracted $i_{\text {corr }}$ as the Protection Current. After understanding behaviour of the electrochemical measurement and the resultant Tafel plot, experimental validation
TABLE 2: The weight loss per day for the freely corroding and the protected washer and A36 steel sample in the $0.6 \mathrm{M} \mathrm{NaCl}$ solution.

\begin{tabular}{lccc}
\hline & \multicolumn{3}{c}{ Weight loss (gm/day) } \\
& $\begin{array}{c}\text { Freely } \\
\text { corroding }\end{array}$ & Protected & $\begin{array}{c}\text { Protection } \\
\text { (inhibition) } \\
\text { efficiency }(\eta)\end{array}$ \\
\hline Washer & 0.00252 & 0.00044 & $83 \%$ \\
A36 steel & 0.00385 & 0.00068 & $82 \%$ \\
\hline
\end{tabular}

of the extracted $i_{\text {corr }}$ for the current-sourced protection system is done. As described in the experimental set-up in the previous section, the metal samples are weighted before and after they are protected using the current-sourced $\left(i_{\text {corr }^{-}}\right.$ based) system. First, the samples are tested for 24 hours (see Figure 6(a)), which is followed by a long-term test (see Figure 6(b)).

During the first experiment (Figure 6(a)), the $i_{\text {corr }}$-based current-sourced protection system shows very good protection behaviour. Both metal samples show more than $82 \%$ efficiency of the proposed protection after 24 hours (see Table 2). The freely corroding washer and the A36 metal sample lose $0.00252 \mathrm{gm} /$ day and $0.00385 \mathrm{gm} /$ day weight, respectively. On the other hand, the protected washer and the A36 steel sample only lose $0.00044 \mathrm{gm} /$ day and $0.00068 \mathrm{gm} /$ day weight, respectively. This results in $83 \%$ and $82 \%$ reduction 
in weight loss in a day for the washer and the A36 steel samples, respectively. These results clearly validate the corrosion protection principle proposed in this paper. The percentages of weight loss for the A36 sample and the washer are closely related. The reason behind such a similarity is related to the type of corrosive medium used for both cases in our experiments.

For the long-term test (see Figure 6(b)), the protected metal samples showed significant reduction in weight loss in comparison to the freely corroding metal. The total weight loss after 16 days for the freely corroding washer and the A36 sample is $0.04032 \mathrm{gm}$ and $0.0616 \mathrm{gm}$, respectively. On the other hand, the total weight loss after 16 days for the protected washer and the A36 sample is $0.00704 \mathrm{gm}$ and $0.01088 \mathrm{gm}$, respectively. Hence, the reduction in the weight loss for the protected samples is again more than $82 \%$ for both metal samples. In the field environment, $82 \%$ protection efficiency may not be adequate to protect the target metal structure for a very long period of time. However, the proposed method will be optimized in the future to provide improved performance and higher protection efficiency. Additionally, the weight loss for the freely corroding samples as well as for the protected samples is linear for the full length of the test period. It is also important to note that all the samples show repeatable behaviour even during the weekends when they are not weighted or corrected during the test. It is also important to note that these tests are performed in a highly corrosive medium $(0.6 \mathrm{M} \mathrm{NaCl}$ solution) and slight deviation in the extraction of the protection current may result in failure of the whole protection system. However, the proposed technique quickly adjusts to the changes and protects the samples effectively.

5.2. Validation of the Functional Adaptive Loop. After validating the principle of $i_{\text {corr }}$-based current-sourced corrosion protection system, the subsequent approach is to implement the automated system including the feedback loop to correct the corrosion protection current $\left(i_{\text {corr }}\right)$. It should be noted that the corrosion status measurement and the protection parameter adjustment are done at every 24 -hour interval. In the future, this adjustment interval can be optimized for more consistent and repeatable performance. To validate the adaptive (feedback) loop for the proposed ACPS, the A36 steel samples in $0.6 \mathrm{M} \mathrm{NaCl}$ solution are tested for 15 days.

The weight loss for the freely corroding and the protected sample is measured every 24 hours and plotted (see Figure 7).

Similar to the previous experiment with the metal washer and the A36 steel sample (Figures 6(a) and 6(b)), the adaptive loop based corrosion protection (Figure 7) works successfully and protects the A36 metal sample using the automated measurement and extraction system described in the previous experimental section. The weight loss of the protected sample and the freely corroding sample after 15 days is $0.01 \mathrm{gm}$ and $0.0574 \mathrm{gm}$, respectively. Hence, the protection (inhibition) efficiency for the adaptively protected sample is $87 \%$, which is better than the results obtained in the manually experimented system in the previous section. These results clearly indicate that the adaptive feedback loop of the proposed ACPS performs effective corrosion protection.

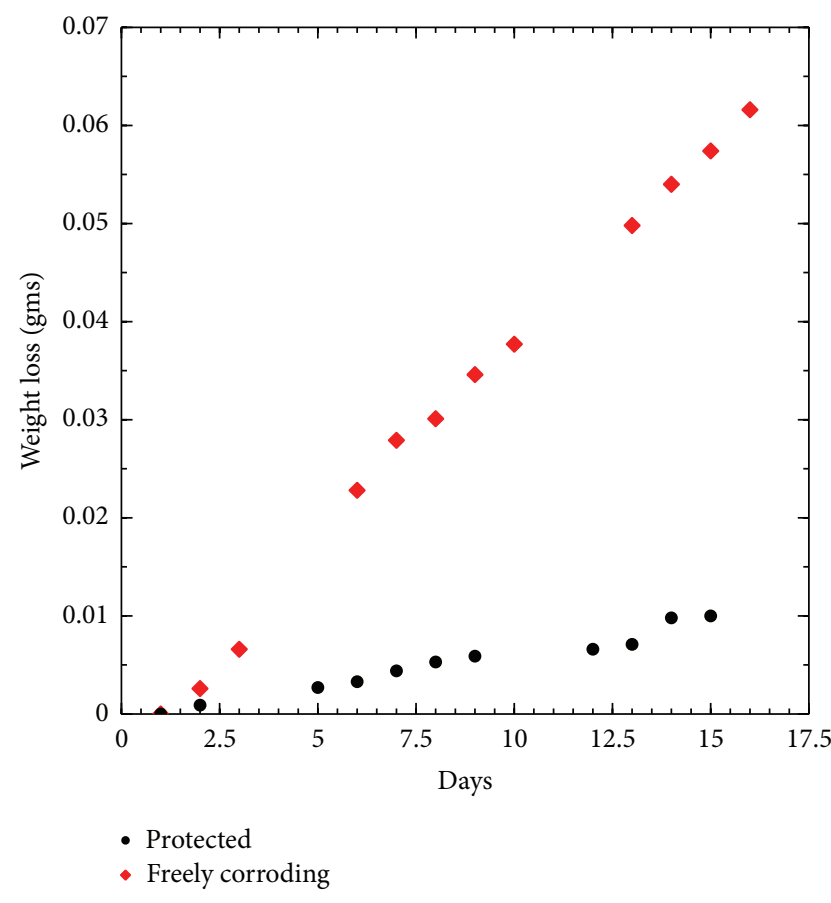

FIGURE 7: The weight loss of the freely corroding and the ACPS protected A36 steel samples in the $0.6 \mathrm{M} \mathrm{NaCl}$ solution for 15 days. Two A36 samples, one corrosion protected using the proposed system and the other freely corroding, are shown. The freely corroding sample lost $0.0616 \mathrm{gm}$ weight and the protected sample lost only 0.01 gm weight.

5.3. ACPS Performance with Different Corrosive Mediums. In the previous subsection, the functionality of the adaptive feedback loop to actively protect the metal from corrosion is discussed. To further validate the proposed principle and the ACPS, the A36 steel samples are tested with different corrosive mediums as mentioned earlier. The individual results for the different corrosive mediums and a comparative chart of the corrosive mediums tested in our laboratory are shown in Figure 8.

From the charts of the different corrosive mediums for roughly two weeks (see Figure 8), it is clearly seen that the weight of the freely corroding metal is linearly decreasing which clearly shows degradation of the A36 steel sample in all corrosive mediums. However, the weight loss and hence the degradation of the metal are not present or minimal for the metal samples that are protected using the proposed ACPS. The weight loss per day for the freely corroding and the ACPS protected metal samples with the different corrosive mediums is also tabulated (see Table 3) and plotted in a clustered column chart (see Figure 8(e)).

The average weight loss per day for the freely corroding metal samples in the $\mathrm{NaCl}$ solution, the sink water solution, the humic acid solution, the wet soil bed, and the dry sand bed is $0.00560 \mathrm{gm}, 0.00784 \mathrm{gm}, 0.00752 \mathrm{gm}$, $0.00602 \mathrm{gm}$, and $0.00003 \mathrm{gm}$, respectively. On the other hand, the average weight loss per day for the ACPS protected samples in the $\mathrm{NaCl}$ solution, the sink water solution, 
TABLE 3: The average weight loss per day for the freely corroding and the ACPS protected A36 steel sample in different corrosive mediums.

\begin{tabular}{lcccc}
\hline Corrosive medium & \multicolumn{2}{c}{$\begin{array}{c}\text { Average weight loss per day } \\
\text { ACPS protected }\end{array}$} & $\begin{array}{c}\text { Protection (inhibition) } \\
\text { efficiency }(\eta)\end{array}$ & Increased life expectancy \\
\hline $0.6 \mathrm{M} \mathrm{NaCl}$ solution & 0.00560 & 0.00071 & $87 \%$ & 7.89 \\
Sink water solution & 0.00784 & 0.00051 & $93 \%$ & 15.37 \\
$50 \mathrm{mg} / \mathrm{L}$ humic acid & 0.00752 & 0.00004 & $99 \%$ & 188.00 \\
solution & 0.00602 & -0.00063 & $\geq 100 \%$ & - \\
Wet soil bed & 0.00003 & 0.00002 & $40 \%$ & 2.50 \\
Dry sand bed & & & \\
\hline
\end{tabular}

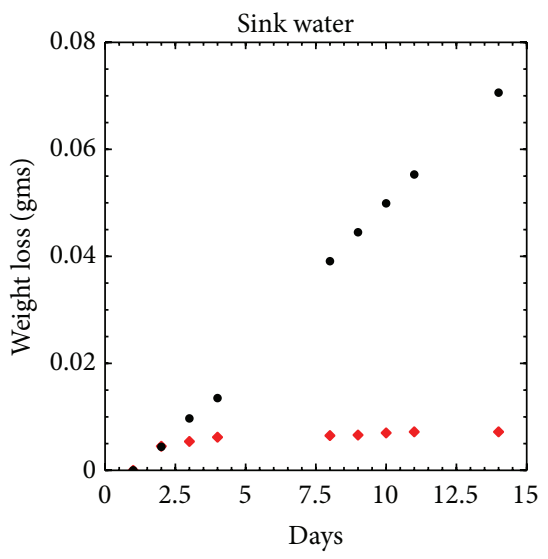

- Freely corroding

- Protected

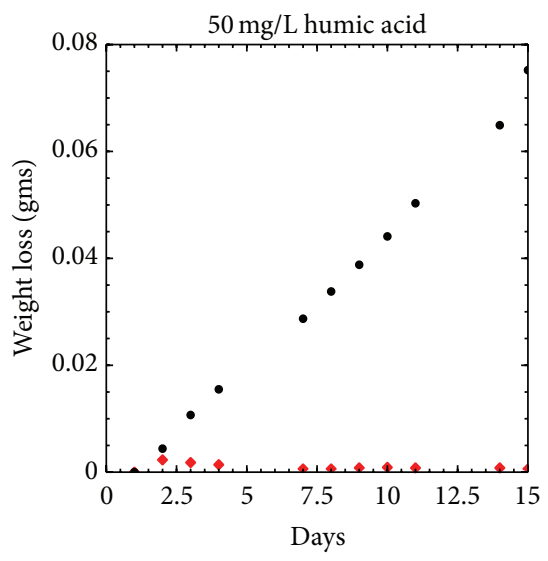

- Freely corroding

- Protected

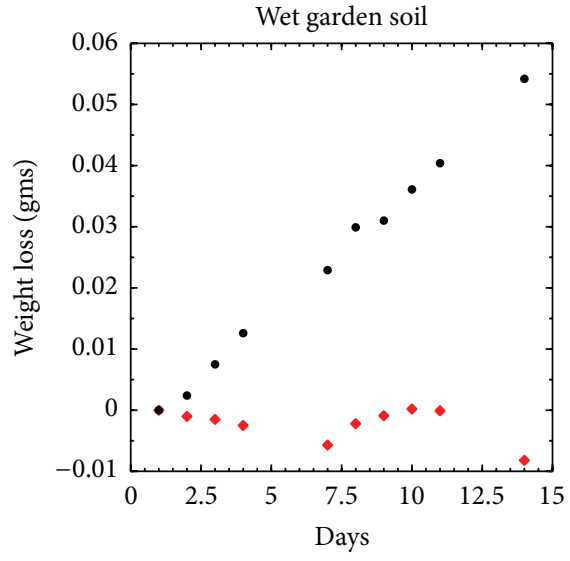

- Freely corroding

- Protected

(a)

(b)

(c)

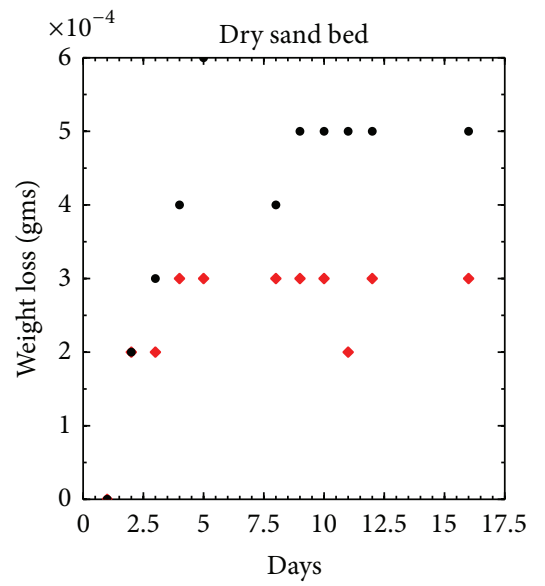

- Freely corroding

- Protected

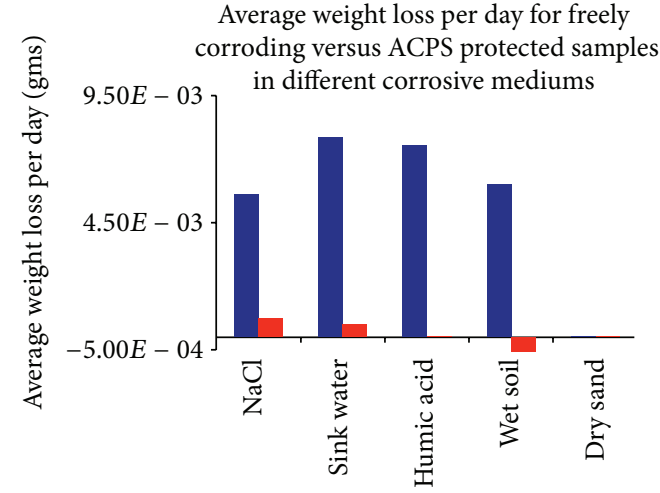

Freely corroding ACPS protected

(e)

Figure 8: The weight loss of the freely corroding and the protected metal samples for more than two weeks in (a) the sink water solution, (b) the $50 \mathrm{mg} / \mathrm{L}$ humic acid solution, (c) the wet soil bed, and (d) the dry sand bed. Two A36 steel samples, one freely corroding and the other protected, are tested in each corrosive medium. (a) The weight loss of the freely corroding and the protected samples in the sink water solution for 13 days. (b) The weight loss of the freely corroding and the protected samples in the $50 \mathrm{mg} / \mathrm{L}$ humic acid solution for 14 days. (c) The weight loss of the freely corroding and the protected samples in the wet soil bed for 14 days. (d) The weight loss of the freely corroding and the protected samples in the dry sand bed for 12 days. (e) The calculated average weight loss per day for the freely corroding and the protected samples in all corrosive mediums. 
the humic acid solution, the wet soil bed, and the dry sand bed is $0.00071 \mathrm{gm}, 0.00051 \mathrm{gm}, 0.00004 \mathrm{gm},-0.00063 \mathrm{gm}$, and $0.00002 \mathrm{gm}$, respectively. The resulting protection (inhibition) efficiency and extension in the life expectance of the target metal are tabulated in Table 3.

As we can see from Table 3 and Figure 8, the ACPS protected sample in the wet soil bed gains slight weight during the experiment. This may be because of the presence of particles or mineral deposits in the garden soil which are attached to the metal sample during the test. However, the freely corroding sample in the same environment (wet soil) linearly loses weight during the experimental period. From the experimental data, the dry sand bed showed minimal weight loss even with the freely corroding sample compared to the other corrosive mediums, which is expected because the dry sand is a poorly corrosive environment. The total weight loss of the freely corroding sample in the sand bed is about two orders of magnitude lower than the other corrosive mediums we test. Additionally, the weight loss for these samples is well within the resolution of the weighting scale and hence the weight loss data is very susceptible to error and incorrect protection (inhibition) efficiency. However, the proposed ACPS reduces metal degradation by $40 \%$. The metal degradation behaviour in the other known lab environments (the $\mathrm{NaCl}$ solution, the humic acid solution, and the sink water solution) shows significant improvement with the ACPS protected system which reduces metal degradation from $87 \%$ to $99 \%$ which represents an accurate performance of the proposed system. It is also important to note that the protection (inhibition) efficiency can be improved even further using frequent corrections compared to $24 \mathrm{hrs}$ used in our study.

In summary, the proposed ACPS works efficiently to reduce the metal degradation in the various corrosive environments. The proposed system is also tested using different metals (Figure 6) to validate the functionality and repeatability of the $i_{\text {corr }}$-based current-sourced ACPS. In the future, even better precision and performance improvement can be obtained by vigorous engineering implementation and software control.

\section{Conclusions}

A method of impressed current cathodic protection (ICCP) using a current-based approach as opposed to the traditional potential-based approach which is the use of a currentcontrolled source over a voltage-controlled source is presented.

To determine and calculate the optimum power and hence the effective protection current $\left(i_{\text {corr }}\right)$, the Tafel plot of the target metal (such as an A36 metal sample) is obtained. The protection current from the Tafel plot is extracted and used to protect a common hardware washer and the A36 steel sample. Using the protection system, the washer and the A36 steel sample show $83 \%$ and $82 \%$ protection (inhibition) efficiency for the target metals.

To further optimize the protection parameter extraction, the experimental automated system is implemented using the LabVIEW VI on a personal computer (PC). The automated adaptive loop is tested using the A36 steel samples in $0.6 \mathrm{M}$ $\mathrm{NaCl}$ solution and the protection (inhibition) efficiency is $87 \%$.

The ACPS is also validated in various corrosive mediums such as the sink water solution, the $50 \mathrm{mg} / \mathrm{L}$ humic acid solution, the wet soil bed, and the dry sand bed. The ACPS protection (inhibition) efficiency for the target metal is $93 \%$, $99 \%, 110 \%$, and $40 \%$ in the sink water, the humic acid, the wet soil bed, and the dry sand bed, respectively. Hence, the life expectancy of the target metal is increased by 7.89 times, 15.37 times, 188 times, and 2.5 times for the sink water, the humic acid, the wet soil bed, and the dry sand bed, respectively.

In summary, traditional cathodic protection applies a fixed potential to the target sample. If the sample's environmental or protection conditions change, the sample will be underprotected or overprotected, which can be detrimental. The new ACPS based approach adjusts the applied cathodic potential regularly by adjusting a constant current $\left(i_{\text {corr }}\right)$, which can achieve the best protection for the sample.

\section{Competing Interests}

The authors declare that they have no competing interests.

\section{Acknowledgments}

The authors are thankful to Mitacs, Canada Research Chairs Program, and Natural Sciences and Engineering Research Council for their financial support to carry out this experimental work at the Simon Fraser University in Burnaby, British Columbia.

\section{References}

[1] P. Atkins and J. de Paula, Physical Chemistry for the Life Sciences, W. H. Freeman and Company, New York, NY, USA, 2005.

[2] M. G. Stewart and D. V. Rosowsky, "Structural safety and serviceability of concrete bridges subject to corrosion," Journal of Infrastructure Systems, vol. 4, no. 4, pp. 146-155, 1998.

[3] J. S. Kong and D. M. Frangopol, "Life-cycle reliability-based maintenance cost optimization of deteriorating structures with emphasis on bridges," Journal of Structural Engineering, vol. 129, no. 6, pp. 818-828, 2003.

[4] D. M. Frangopol, K.-Y. Lin, and A. C. Estes, "Reliability of reinforced concrete girders under corrosion attack," Journal of Structural Engineering, vol. 123, no. 3, pp. 286-297, 1997.

[5] A. A. Torres-Acosta and M. Martínez-Madrid, "Residual life of corroding reinforced concrete structures in marine environment," Journal of Materials in Civil Engineering, vol. 15, no. 4, pp. 344-353, 2003.

[6] D. M. Frangopol, K.-Y. Lin, and A. C. Estes, "Life-cycle cost design of deteriorating structures," Journal of Structural Engineering, vol. 123, no. 10, pp. 1390-1401, 1997.

[7] C. G. Soares, Y. Garbatov, A. Zayed, and G. Wang, "Influence of environmental factors on corrosion of ship structures in marine atmosphere," Corrosion Science, vol. 51, no. 9, pp. 2014-2026, 2009. 
[8] H. P. Leckie and H. H. Uhlig, "Environmental factors affecting the critical potential for pitting in 18-8 stainless steel," Journal of the Electrochemical Society, vol. 113, no. 12, pp. 1262-1267, 1966.

[9] K. Suzumura and S.-I. Nakamura, "Environmental factors affecting corrosion of galvanized steel wires," Journal of Materials in Civil Engineering, vol. 16, no. 1, pp. 1-7, 2004.

[10] J. J. S. Rodríguez, F. J. S. Hernández, and J. E. G. González, "The effect of environmental and meteorological variables on atmospheric corrosion of carbon steel, copper, zinc and aluminium in a limited geographic zone with different types of environment," Corrosion Science, vol. 45, no. 4, pp. 799-815, 2003.

[11] L. V. Delinder, An Introduction to Corrosion, Corrosion Basics, NACE, Houston, Tex, USA, 1984.

[12] C. Chunan, "Corrosion electrochemistry," Journal of Chemistry Industry, 1994.

[13] D. A. Vermilyea and C. S. Tedmon," "A simple crevice corrosion theory," Journal of the Electrochemical Society, vol. 117, no. 4, pp. 437-440, 1970.

[14] Z. P. Bazant, "Physical model for steel corrosion in concrete sea structures-theory," Journal of the Structural Division, vol. 105, no. 6, pp. 1137-1153, 1979.

[15] R. G. Kelly Jr., Electrochemical Techniques in Corrosion Science and Engineering, Mercel Dekker, Basel, Switzerland, 2003.

[16] G. A. Jacobson, Corrosion 101, NACE, Houston, Tex, USA, 2014, http://www.nace.org/.

[17] B. Wessling and J. Posdorfer, "Corrosion prevention with an organic metal (polyaniline): corrosion test results," Electrochimica Acta, vol. 44, no. 12, pp. 2139-2147, 1999.

[18] E. Heitz, "Mechanistically based prevention strategies of flowinduced corrosion," Electrochimica Acta, vol. 41, no. 4, pp. 503509, 1996.

[19] C. Christodoulou, G. Glass, J. Webb, S. Austin, and C. Goodier, "Assessing the long term benefits of Impressed Current Cathodic Protection," Corrosion Science, vol. 52, no. 8, pp. 26712679, 2010.

[20] H. Park, K. Y. Kim, and W. Choi, "Photoelectrochemical approach for metal corrosion prevention using a semiconductor photoanode," Journal of Physical Chemistry B, vol. 106, no. 18, pp. 4775-4781, 2002.

[21] X. G. Zhang and E. M. Valeriote, "Galvanic protection of steel and galvanic corrosion of zinc under thin layer electrolytes," Corrosion Science, vol. 34, no. 12, pp. 1957-1972, 1993.

[22] M. Morcillo, R. Barajas, S. Feliu, and J. M. Bastidas, "A SEM study on the galvanic protection of zinc-rich paints," Journal of Materials Science, vol. 25, no. 5, pp. 2441-2446, 1990.

[23] J. B. Bushman, Impressed Current Cathodic Protection System Design, Bushman \& Associates, 2010.

[24] S. Szabó and I. Bakos, "Impressed current cathodic protection," Corrosion Reviews, vol. 24, no. 1-2, pp. 39-62, 2006.

[25] L. Y. Xu, X. Su, and Y. F. Cheng, "Effect of alternating current on cathodic protection on pipelines," Corrosion Science, vol. 66, pp. 263-268, 2013.

[26] S. D. Cramer, B. S. Covino Jr., S. J. Bullard et al., "Corrosion prevention and remediation strategies for reinforced concrete coastal bridges," Cement and Concrete Composites, vol. 24, no. 1, pp. 101-117, 2002.

[27] R. W. Revie, Corrosion and Corrosion Control, John Wiley \& Sons, Hoboken, NJ, USA, 2008.
[28] A. Oni, "Effects of cathodic overprotection on some mechanical properties of a dual-phase low-alloy steel in sea water," Construction and Building Materials, vol. 10, no. 6, pp. 481-484, 1996.

[29] J. J. Chang, "A study of the bond degradation of rebar due to cathodic protection current," Cement and Concrete Research, vol. 32, no. 4, pp. 657-663, 2002.

[30] S. Amami, C. Lemaitre, A. Laksimi, and S. Benmedakhene, "Characterization by acoustic emission and electrochemical impedance spectroscopy of the cathodic disbonding of $\mathrm{Zn}$ coating," Corrosion Science, vol. 52, no. 5, pp. 1705-1710, 2010.

[31] P. Pedeferri, "Cathodic protection and cathodic prevention," Construction and Building Materials, vol. 10, no. 5, pp. 391-402, 1996.

[32] Underground Storage Tank Branch and Mississippi Department of Environmental Quality, "Guidelines for the evaluation of underground storage tank cathodic protection systems," July 2002, http://www.neiwpcc.org/neiwpcc_docs/ MS\%20DEQ\%20Guidelines\%20for\%20the\%20Evaluation \% 20of\%20UST\%20Cathodic\%20Protection\%20Systems.pdf.

[33] T. Kodama, K. Kimura, Y. Shinoda, and N. Mochizuki, "Determination of instant-off potential of cathodically protected reinforcing steel in concrete bridges located in coastal atmosphere," in Proceedings of the CORROSION, NACE International, San Diego, Calif, USA, March 2006.

[34] L. V. Nielsen, K. V. Nielsen, B. Baumgarten, H. BreuningMadsen, and H. Rosenberg, "AC induced corrosion in pipelines: detection, characterization and mitigation," in Proceedings of the CORROSION, NACE International, New Orleans, La, USA, March-April 2004.

[35] F. Kajiyama and Y. Nakamura, "Effect of induced alternating current voltage on cathodically protected pipelines paralleling electric power transmission lines," Corrosion, vol. 55, no. 2, pp. 200-205, 1999.

[36] J. Ansuini Frank and J. R. Dimond, "Field tests on an advanced cathodic protection coupon," CORROSION/2005 paper 039, 2005.

[37] N. G. Thompson and J. A. Beavers, "Measurements of IR-drop free pipe-to-soil potentials on buried pipelines," in The Measurement and Correction of Electrolyte Resistance in Electrochemical Tests, vol. 1056, p. 168, ASTM International, 1990.

[38] B. S. Jang and B. H. Oh, "Effects of non-uniform corrosion on the cracking and service life of reinforced concrete structures," Cement and Concrete Research, vol. 40, no. 9, pp. 1441-1450, 2010.

[39] Y. Zhao, A. R. Karimi, H. S. Wong, B. Hu, N. R. Buenfeld, and W. Jin, "Comparison of uniform and non-uniform corrosion induced damage in reinforced concrete based on a Gaussian description of the corrosion layer," Corrosion Science, vol. 53, no. 9, pp. 2803-2814, 2011.

[40] F. Mansfeld, "Tafel slopes and corrosion rates from polarization resistance measurements," Corrosion, vol. 19, no. 10, pp. 397402, 1973.

[41] Z. Shi, M. Liu, and A. Atrens, "Measurement of the corrosion rate of magnesium alloys using Tafel extrapolation," Corrosion Science, vol. 52, no. 2, pp. 579-588, 2010.

[42] A.-H. J. Al-Tayyib, Mohammed, and S. Khan, "Corrosion rate measurements of reinforcing steel in concrete by electrochemical techniques," ACI Materials Journal, vol. 85, no. 3, pp. 172-177, 1988. 
[43] E. McCafferty, "Validation of corrosion rates measured by the Tafel extrapolation method," Corrosion Science, vol. 47, no. 12, pp. 3202-3215, 2005.

[44] F. Bentiss, M. Traisnel, L. Gengembre, and M. Lagrenée, "New triazole derivative as inhibitor of the acid corrosion of mild steel: electrochemical studies, weight loss determination, SEM and XPS," Applied Surface Science, vol. 152, no. 3, pp. 237-249, 1999. 

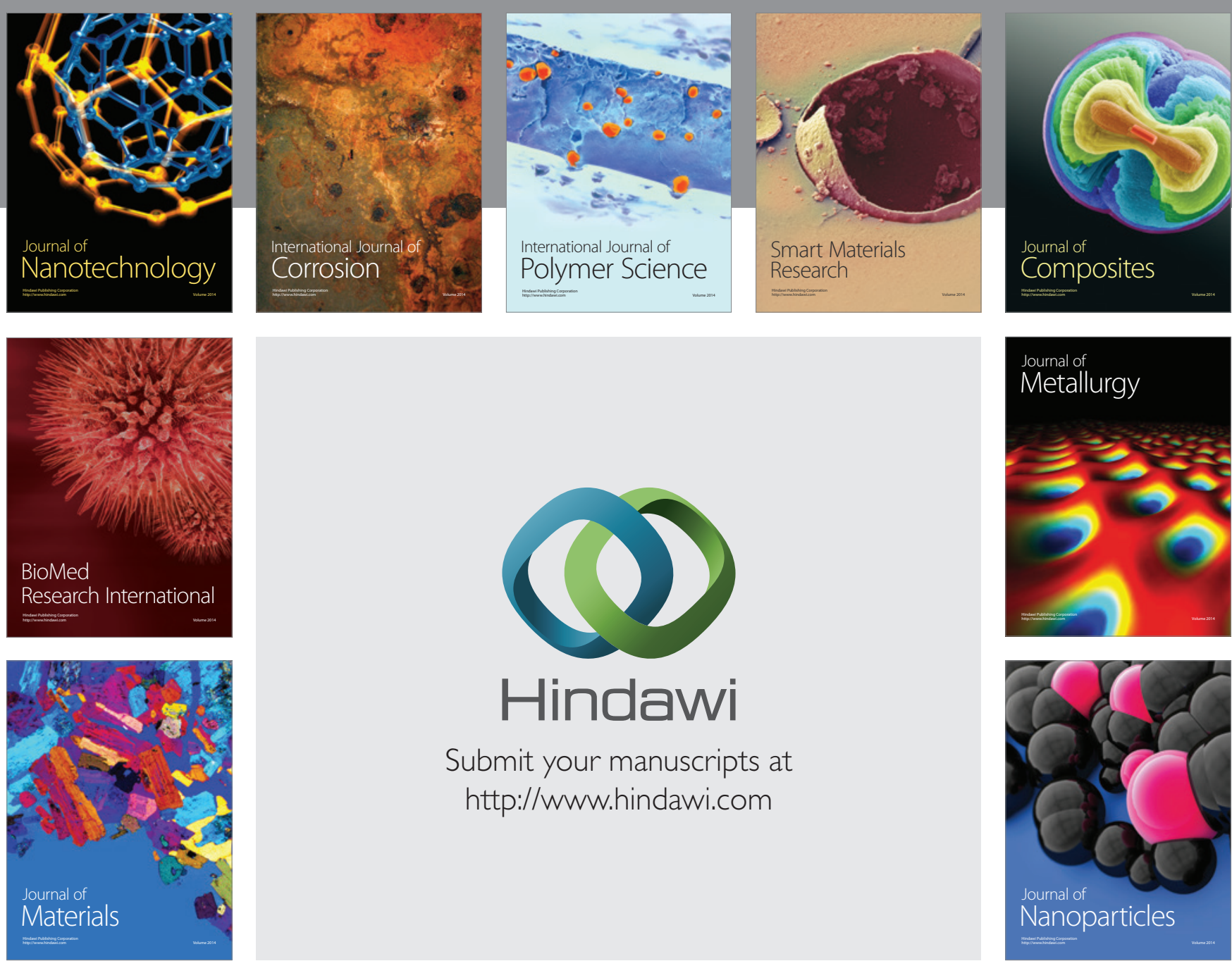

\section{Hindawi}

Submit your manuscripts at

http://www.hindawi.com

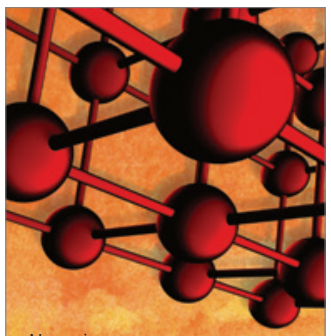

Materials Science and Engineering
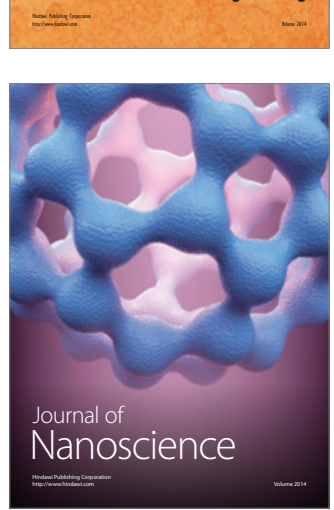
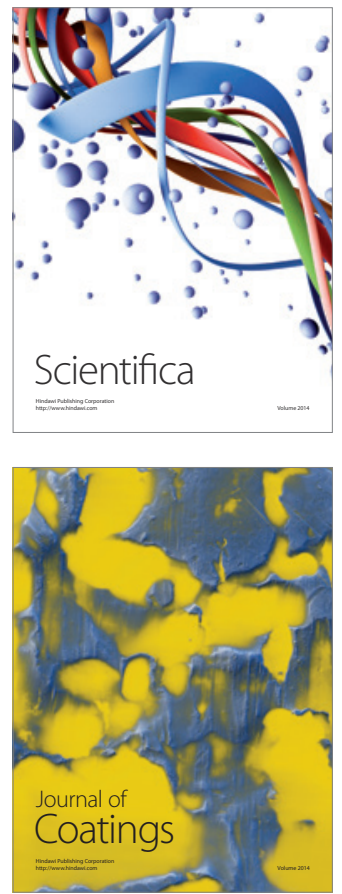
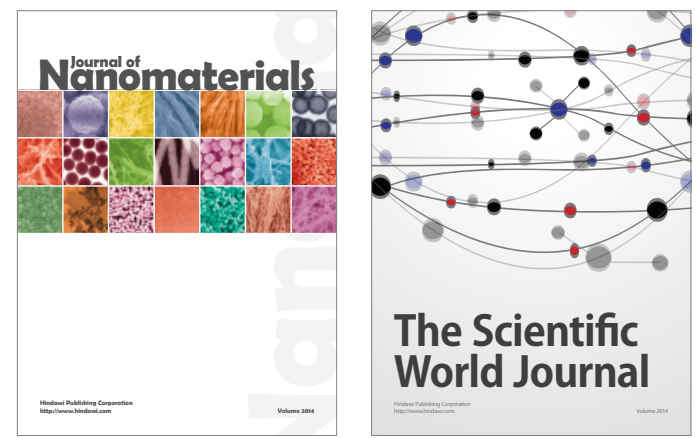

The Scientific World Journal
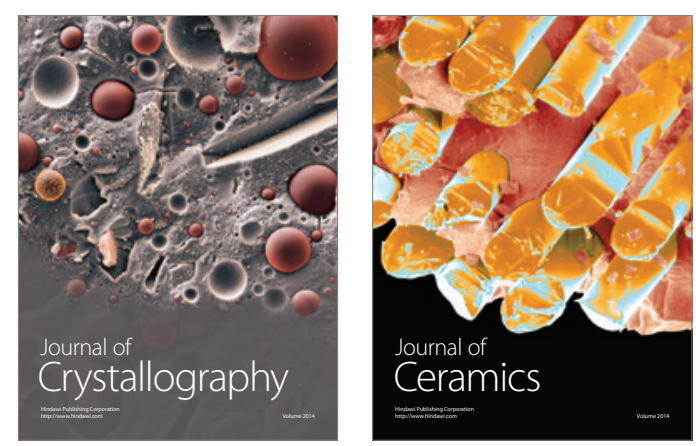
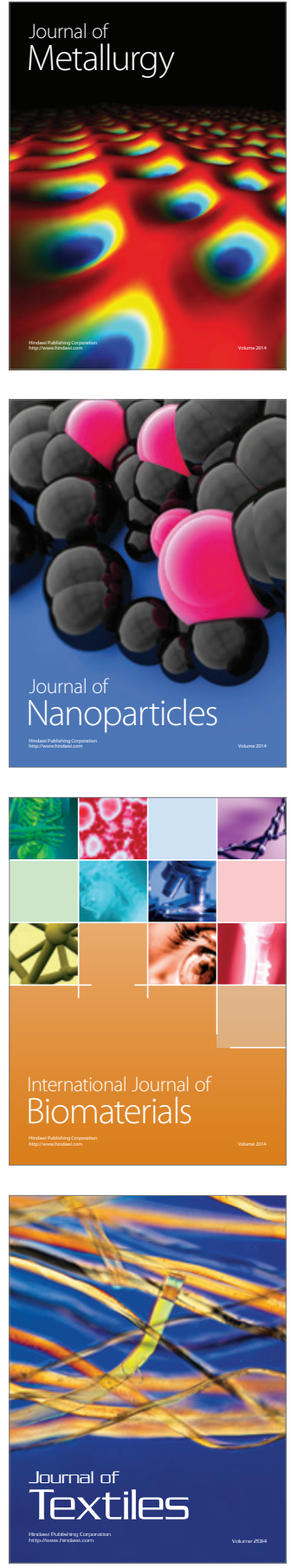